\title{
Further evidence for an intermittent pattern of neural tube closure in humans
}

\author{
Mary J Seller
}

\begin{abstract}
Evidence is presented to support the recent suggestion that human neural tube closure is similar to that observed in mice, and comprises several regionally distinct closure sites rather than being a simple zipping up process. Seven subjects, each with more than one neural tube defect (NTD), are described. Comparative studies of the location of the lesions in relation to the closed parts of the neural tube imply that such NTD could only have come about if there were intermittent closure of the neural tube.
\end{abstract}

(f Med Genet 1995;32:205-207)

The recent suggestion that, during embryogenesis, human neural tube closure does not involve a simple "zipping up" process but rather follows an intermittent pattern akin to that observed in mice ${ }^{12}$ has yet to be proven. In mice, four distinct closure regions have been defined, numbered 1 to 4 . The initial closure is at the cervical/hindbrain boundary which is the future mid-cervical region, and proceeds from there bidirectionally, rostral closure 1 into the head and caudal closure 1 down the body. Closure 2 in the head is the second de novo closure initiation site and begins at the prosencephalon-mesencephalon (forebrain/midbrain) boundary; again, it is bidirectional. Closure 3 initiates closure at the cranial extremity of the neuraxis. It starts at the stomodaeum and is one way, travelling upwards to meet the descending rostral limb of closure 2 . The area between closures 2 and 3 represents the true anterior neuropore. Closure 4 is over the rhombencephalon. ${ }^{134}$ It is not a true initiation closure site: rather, it completes closure of the cranial neural tube and constitutes the second, or posterior, cranial neuropore. Van Allen et al explained, most logically, the origin of human cranial and thoracic neural tube defects (NTD) on the basis of these four closure sites, thus providing circumstantial evidence for their existence in humans. They also added to the list a fifth closure region at the extreme caudal end of the neural tube to account for lower spinal NTD which they propose travels rostrally to meet the caudal closure 1 . The area between closures 1 and 5 comprises the posterior neuropore. Closure 5 may or may not also involve the canalisation process which extends the neural tube distally subcutaneously after the completion of primary neurulation. ${ }^{5}$

The prospects of directly proving the in- termittent pattern of neural tube closure in humans seems remote, and so proof will rather have to be deduced from observations of particular NTD types, the most salient points probably emerging from a study of rare and unusual NTD lesions, especially multiple forms in a single person. One possible case has been presented by Busam et al, ${ }^{6}$ although it was complicated by the presence of adherent amnion. I report here seven human fetuses with more than one NTD which I believe contribute to an understanding of the situation.

\section{Materials and methods}

Detailed case histories are omitted. One case aborted spontaneously, and the remainder were detected during routine prenatal diagnosis in the South East Thames Regional Genetics Centre at Guy's Hospital, London, incorporating some or all of: maternal serum alphafetoprotein (AFP) testing, amniocentesis with amniotic fluid AFP estimation and acetylcholinesterase studies, and ultrasonography. The parents were all counselled as to the diagnosis and prognosis, and all elected to terminate the pregnancies.

The fetuses were received for postmortem examination and each was considered to be non-syndromal, and thus regarded as of multifactorial aetiology. The relevant findings relating to neural tube closure in each fetus are as follows, and are shown schematically in fig 1. For clarity other details are omitted.

Case 1 was a 17 week male fetus with anencephaly of the meroacrania type with the occipital bone and foramen magnum intact (fig 2 ). There was also an open spina bifida of the entire sacrum.

Case 2 was a male of 20 weeks in whom there was anencephaly of the holoacrania type, that is, also involving the occipital bone and foramen magnum. The second lesion was a lumbosacral spina bifida extending from L1 to S5, with most of the sacral part covered by skin.

Case 3 was a male of 18 weeks with holoacrania type anencephaly continuous with complete cervical spina bifida (fig 3). The neck was short and the cervical vertebrae reduced. The upper thoracic vertebrae were malformed and there was marked scoliosis, but there was no spina bifida. However, from $\mathrm{T} 10$ to $\mathrm{S} 5$ there was spina bifida, which was open from T10 to L5 and closed by full thickness skin from $\mathrm{S} 1$ to S5. 


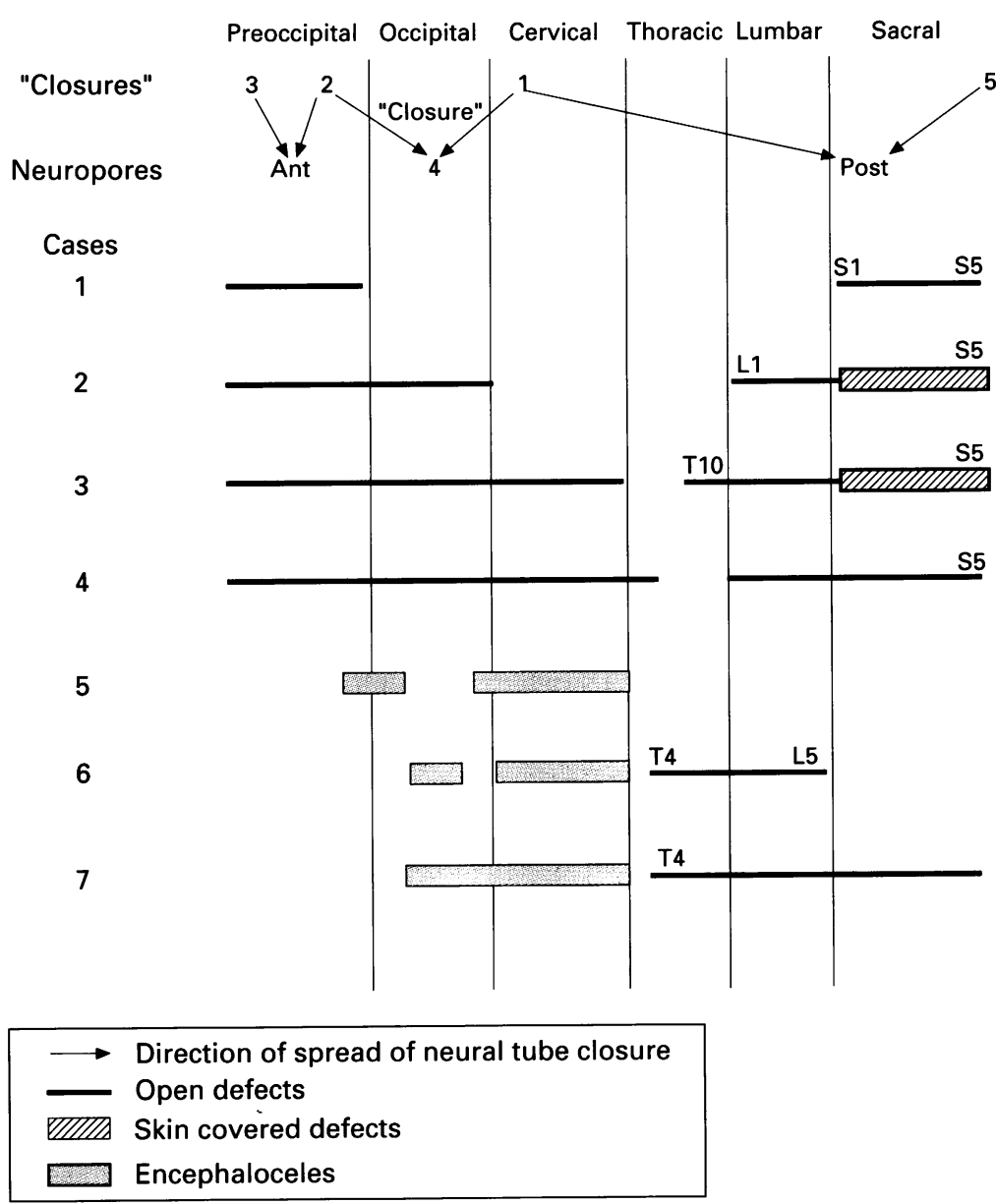

Figure 1 Diagrammatic respresentation of lesions observed in each fetus and their relation to the proposed intermittent pattern of neural tube closure in humans.
Case 4 aborted spontaneously at 16 weeks but was severely macerated and corresponded to around 9 weeks in size and development. There was a continuous rostral lesion comprising holoacrania and cervical and upper thoracic spina bifida. The neural tube was closed for a short distance in the lower thoracic region. Then there was another open spina bifida through the lumbar region to the lower sacrum.

Case 5 was a 21 week female in whom the lesions were restricted to the head and neck (fig 4). There was microcephaly and two separate, but closely apposed, meningoencephaloceles of moderate size, the more rostral being the larger. Both were covered by full thickness hairy skin proximally, but this soon changed to thin skin over most of the sacs. There were two separate underlying bony lesions. The most cranial was a circular lesion $(1.2 \mathrm{~cm}$ anteroposterior, $1 \mathrm{~cm}$ across) in the midline at the parieto-occipital junction. The lesion largely affected the parietal bones but the anterior margin of the occipital bone was also involved. The occipital bone was then complete in the midline for a distance of $6 \mathrm{~mm}$ until it was interrupted by another oval shaped lesion in the midline $(1.7 \mathrm{~cm}$ anteroposterior, $1 \mathrm{~cm}$ laterally). Inferior to this was a $2 \mathrm{~mm}$ connective tissue bridge. The foramen magnum was essentially absent and this lesion was continuous with a spina bifida involving all of the cervical vertebrae which were reduced; the neck was very short. The remainder of the spine was normally formed.
Figure 2 Case 1: meroacrania and sacral spina bifida.
Figure 3 Case 3: holoacrania with cervical Figure 3 Case 3: holoacrania with cervical occipital meningoencephal
spina bifida and thoracolumbosacral spina bifida. meningoencephalocele.

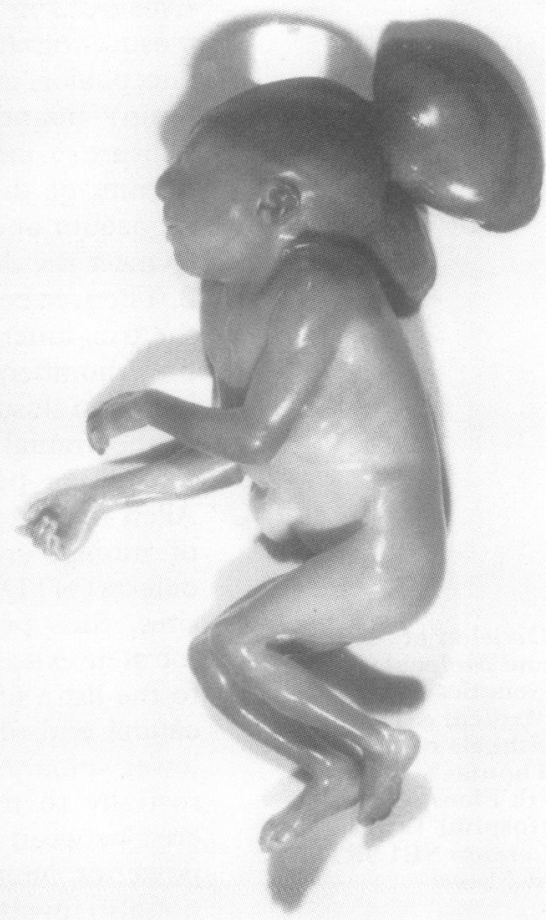

Figure 4 Case 5: microcephaly with a parietooccipital meningoencephalocele and an occipital 


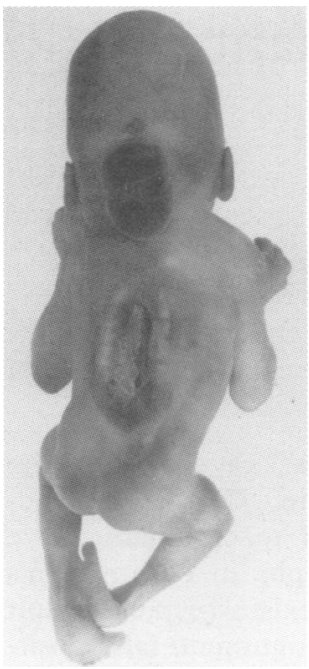

Figure 5 Case 6: small occipital meningocele, cervical meningocele, and a thoracolumbar spina bifida.
Case 6 was a 20 week male fetus whose mother and maternal brother have spina bifida cystica. There were three separate lesions: a cranial meningocele ( $1 \mathrm{~cm}$ diameter), a larger cervical meningocele, and a thoracolumbar spina bifida cystica (fig 5). The parietal bones and the parieto-occipital junction were all intact. The occipital bone was hypoplastic, and there was a small (around 1 to $2 \mathrm{~mm}$ ) lesion in the midline of the occipital bone, just superior to the foramen magnum which was complete. The cervical vertebrae were reduced and the neck was short; there was a total spina bifida. The vertebral arches of the upper thoracic spine had fused but the vertebrae were malformed. There was rib fusion and there was scoliosis to the right. There was an open spina bifida from T4 to L5. The sacral spine was closed.

Case 7 was a female of 22 weeks with a median occipital encephalocele covered with full thickness skin and a thoracolumbosacral spina bifida. There was a midline lesion in the occipital bone and a cervical spina bifida covered by full thickness skin. The upper thoracic spine was intact, but from around T4 to the lower sacrum there was an open spina bifida.

\section{Discussion}

These cases are discussed in the light of the multiple closure sites described in mice ${ }^{1}$ and as proposed for humans by Van Allen et al. ${ }^{2}$ The relationships between the cases and the closure sites are also shown in fig 1 .

In principle, if there is more than one NTD in a person it would suggest that there might well be an intermittent pattern of closure. Cases 1 to 4 , with separate lesions at the rostral and caudal ends of the neural tube, however, do not immediately appear to support the hypothesis of multiple closure sites in the human neural tube. Rather, they could be interpreted on the old "single zipper" model, as failure to close at both ends of a single bidirectional process, which starts in the midcervical region. However, perhaps because frontocranial NTD lesions commonly seen in south east Asia ${ }^{7}$ are so rare in the western world, it is easily overlooked that the true anterior neuropore is in the frontal region of the head, and that closure of the prosencephalon (closure 3 ) is part of primary neurulation. In cases 1 and 2 , there is no facioschisis but there is anencephaly. Closure 3 has therefore occurred while the more caudal closure of the head has not, thus supporting the idea at the very least that there is a separate closure 3 in humans.

The two forms of anencephaly, meroacrania and holoacrania, as seen in cases 1 and 2 respectively, are so consistent in their morphology that it is difficult to dispute that meroacrania is caused by failure of another distinct region of neural tube closure (closure 2), while holoacrania involves failure of closure
2 together with failure of a more caudal region. However, there is no direct proof in these cases.

At the caudal end of the neural tube, the existence of a spinal lesion restricted to the sacrum in case 1, but involving the thoracolumbosacral and lumbosacral regions in cases 3 and 2 respectively, with the sacral parts of both covered by skin, argues for a separate process producing closure of the sacral region, distinct from that involved in closure of the thoracolumbar region. Further evidence for this comes from case 6 where the spinal lesion is confined to the region of T4 and L5 and the sacral spine is normally formed. It would appear that closure 5 does exist, but the location in the neuraxis suggested here (S1-S5) is different from and more distal to that proposed by Van Allen et al. ${ }^{2}$

Cases 5 to 7 strongly support the idea of separate closure sites in the back of the head. In case 5, the local lesion affecting the posterior midline of the parietal bones and the most rostral midline region of the occipital bone agrees with the notion that this area is the junction point of two separate closures. The discrete, lower lesion in the occipital bone, after a narrow normally formed part, together with the absent foramen magnum and continuous with cervical spina bifida, suggests that the proposed closure 4 could itself involve intermittent or perhaps bidirectional closure, rather than being a smooth unidirectional process. Such a closure pattern is also suggested by case 6 where there is a very small local lesion in the lower part of the occipital bone but intact foramen magnum and then a separate cervical spina bifida.

Overall, comparative studies of these fetuses offer support for the notion that during embryogenesis in humans, as in mice, there is regional closure of the neural tube, rather than a continuous "zipping up" process. It is felt that it is difficult to explain the occurrence of at least some of the lesions without such a process. However, many details, such as the direction of closure within each region and the precise location along the neuraxis of each closure region, are still lacking.

1 Golden JA, Chernoff GF. Intermittent pattern of neural tube closure in two strains of mice. Teratology 1993;47: 73-80.

2 Van Allen MI, Kalousek DK, Chernoff GF, et al. Evidence for multi-site closure of the neural tube in humans. $A m \mathcal{F}$ Med Genet 1993;47:723-43.

3 Golden JA, Chernoff GF. Anterior neural tube closure in the mouse: fuel for disagreement with the classical theory. the mouse: fuel for disag

4 Juriloff DM, Harris MJ, Tom C, MacDonald KB. Normal mouse strains differ in the site of initiation of closure of the cranial neural tube. Teratology 1991;44:225-33.

5 Schoenwolf GC. Histological and ultrastructural studies of secondary neurulation in mouse embryos. Am $\mathcal{F}$ Anat 1948;169:361-76.

6 Busam KJ, Roberts DJ, Golden JA. Clinical teratology counselling and consultation case report: two distinct anterior neural tube defects in a human fetus: evidence for an intermittent pattern of neural tube closure. $T e-$ ratology 1993;48:399-403.

$7 \mathrm{Thu} \mathrm{A}$, Kyu H. Epidemiology of frontoethmoidal encephalomeningocoeles in Burma. I Epidemiol Community Health 1984;38:89-98. 